\title{
Convergencias y desencuentros en las ideas de cultura y valores en durkheim y en weber
}

\section{Convergences and Discrepancies in the Ideas of Culture and Values in Durkheim and Weber}

\author{
Paloma Palau Valderrama 24
}

\section{Resumen}

En el presente texto señalo los puntos de encuentro y diferencias fundamentales en las ideas de cultura y valores de los sociólogos clásicos Durkheim y Weber. En primer lugar, expongo las ideas de ambos autores a partir del análisis de su obra en su contexto histórico y en diálogo con relecturas contemporáneas de los dos clásicos, identificando sus sentidos. Subrayo la relación de las nociones de cultura y valores con otras relevantes en el pensamiento de cada uno, tales como moral, valores ideales y civilización en Durkheim; y significación cultural, ethos y racionalización en Weber. Después, apunto las convergencias y desencuentros en las nociones de cultura y valores. Finalmente, argumento que en las teorizaciones de ambos sociólogos clásicos existe la semilla de una perspectiva para comprender la pluralidad axiológica y las tensiones entre sujeto y sociedad en los contextos multiculturales y globalizados contemporáneos.

Palabras clave: sociología clásica. Durkheim. Weber. Cultura.

\section{Abstract}

In this paper I point out some fundamental convergences and differences in the ideas of culture and values of the classical sociologists Durkheim and Weber. In the

\footnotetext{
${ }^{24}$ Doutoranda em Música na Área Etnomusicologia/Musicologia (Programa de Pós-Graduação em Música) da Universidade Federal do Rio Grande do Sul (UFRGS); ORCID: https://orcid.org/00000003-2832-0762; Email: palomapala@gmail.com.
} 
first place, I present the ideas of both authors from the analysis of their work in its historic context and in dialogue with contemporary re-readings of the two classics, identifying their meaning. I emphasize the relationship of the notions of culture and values with other relevant ones in the thought of each one, such as moral, ideal values and civilization in Durkheim; and cultural significance, ethos and rationalization in Weber. Then, I remark the convergences and disagreements in the notions of culture and values. Finally, I argue that in the theorizations of both classical sociologists there is the seed of a perspective to understand the axiological plurality and the tensions between subject and society in contemporary multicultural and globalized contexts.

Keywords: classical sociology. Durkheim. Weber. Culture.

\section{Introducción}

En este texto presento la idea de cultura y su relación con los valores para los sociólogos clásicos Durkheim y Weber basada en algunas de sus obras y destaco sus principales convergencias y desencuentros. Adicionalmente dialogo con algunos análisis del tema hechos por otros autores para discutir tales conceptos. Reviso las obras de Durkheim Las formas elementales de la vida religiosa (2000), Sociología y filosofía (2006) As Regras do Método Sociológico (1978) y algunos planteamientos de La División del Trabajo Social (1987). De Weber, me enfoco en Economía y sociedad (1964), Ensayos sobre metodología sociológica (1958) y A ética protestante e o espíritu do capitalismo (2004).

La temática elegida si bien no es preponderante en la teoría sociológica clásica, su relevancia se deriva de los siguientes puntos. En primer lugar, aunque la noción de cultura no es sobresaliente en los albores de la sociología, es posible identificar algunos de los sentidos de su conceptualización que atraviesan el pensamiento durkhemiano y weberiano, y verificar la cercana conexión establecida entre las ideas de cultura y valores al observar la utilización que hacen de ella ambos sociólogos. En segunda instancia, la cultura se convierte en un elemento 
central en las teorizaciones de las ciencias sociales contemporáneas y otras áreas del conocimiento, y al desarrollarse en varias direcciones, se torna polisémica. No obstante, es pertinente esclarecer que, escapa a los objetivos y al alcance de este trabajo identificar la multiplicidad de caminos que toma la reflexión sobre la cultura y los valores en la última centuria.

Este artículo se estructura en cuatro partes. Le precede una introducción al tema con un sucinto esclarecimiento sobre las connotaciones de los términos en discusión en su contexto histórico. Como expondré en este texto, ambos científicos utilizan las connotaciones usadas en sus respectivos países y convergen en algunos aspectos, como la relevancia y significado cultural de los valores para la configuración de cada sociedad.

La segunda sección explica los desarrollos de Durkheim, comentando su uso de los conceptos de valores ideales y civilización, y la tercera, aborda las teorizaciones de Weber, en paralelo con sus acepciones de significación y ethos. La elaboración de estos dos apartados es una interpretación reflexiva que extrae las explicaciones de la cultura y los valores a partir de los textos citados y los de otros autores que examinaron sus obras.

Para finalizar, expongo las convergencias de ambas propuestas sociológicas y algunos de sus desencuentros. A esta última parte, añado una consideración sobre la importancia de tales concepciones en la contemporaneidad.

\section{Comentarios sobre las nociones de cultura y civilización}

Si bien, tanto los conceptos de cultura como de civilización fueron usados en Francia, Inglaterra y Alemania, donde tuvieron sus desarrollos teóricos desde la modernidad, los sentidos y relevancia variaban de acuerdo con los contextos nacionales. Mientras que en Francia e Inglaterra la idea de civilización es la que guía los preceptos sobre la sociedad occidental, en Alemania la noción de cultura está en el centro de las discusiones.

En Alemania primaba una acepción de cultura particularista influenciada por la idea de la nación y del "alma” del pueblo; las características del lenguaje y desarrollos intelectuales y morales son relevantes en ella. En su tendencia más 
nacionalista de inicio de siglo XX se aproxima a la idea de raza, noción que Weber critica severamente y advierte el uso manipulativo que hace de ella el patriotismo (FREUND, 2003, p. 165). Elías (1994) señala que la idea de cultura en Alemania es pensada de modo específico para hechos religiosos, artísticos e intelectuales. Así, lo cultural se refiere más a los logros y valores de una sociedad específica que al comportamiento de una persona.

En Francia el significado cultura tenía como legado la referencia al desarrollo intelectual y artístico tan exaltados durante la Ilustración, lo que deviene en una acepción de cultura como educación en la siguiente centuria. Era común traducir al francés los textos extranjeros que usaran la palabra de cultura, por la de civilización. Ya a finales de siglo adquiere un matiz universalista que entiende a la humanidad como una unidad (CUCHE, 2002).

Ahora bien, en Francia e Inglaterra la noción de civilización tiene el sentido de progreso de la sociedad occidental, entendida como la más avanzada, y por ende, el desarrollo de la humanidad. Elías capta el sentido expansionista del término civilización, apuntando la relativa estabilidad de los márgenes políticos en ambos países, que no tuvieron mayores preocupaciones con la definición de una identidad nacional y centraron sus objetivos en los propósitos colonizadores bajo el ideal civilizatorio. A diferencia de los alemanes, cuyas fronteras nacionales fueron permanentemente reconstruidas en los conflictos geopolíticos del continente europeo, el asunto de la identidad nacional, o la pregunta por la cultura, se mantuvo presente. En palabras del autor:

Enquanto o conceito de civilização inclui a função de dar expressão a uma tendência continuamente expansionista de grupos colonizadores, o conceito de Kultur [cultura] reflete a consciência de si mesma de uma nação que teve de buscar e constituir incessante e novamente suas fronteiras, tanto no sentido político como espiritual, e repetidas vezes perguntar a si mesma: 'Qual é, realmente, nossa identidade?' (ELÍAS, 1994, p. 25).

En la acepción francesa e inglesa, se considera que una persona puede ser "civilizada", o comportarse dentro de unas convenciones con las que se demuestra su grado de educación y posición en la sociedad, resalta Elías. En contraste, civilización para los alemanes se limita a un valor secundario relativo a la utilidad y 
a la apariencia externa humana, por lo cual para referirse a los individuos, en el mismo sentido que civilizado, se debería usar cultivado. Así, mientras que cultura se refiere al contexto circunscrito de la identidad de una sociedad específica, civilización tiene un sentido de búsqueda de lo universal, restando valor a las diferencias nacionales.

No obstante su proximidad, el desarrollo histórico de las nociones de civilización y cultura las lleva a colocarse en antítesis en ciertos momentos en el contexto alemán como resalta Elías. La lengua francesa era hablada en la corte alemana como sinónimo de educación, estilo y distinción, y por el contrario, el alemán se asociaba a lo burdo, a las clases bajas. La clase media intelectual va a reivindicar el valor de la lengua nacional con el romanticismo, de modo que la antítesis se observa así: civilización se puede asociar a una cortesía falsa, externa del individuo, mientras que cultura se vincula a lo espiritual, a la virtud auténtica e interior (p. 29).

Por otra parte, en el contexto académico, la definición y uso de los términos está en relación íntima con la emergencia y consolidación de la disciplina sociológica a finales del siglo XIX, marcada por el campo de poder científico de cada contexto nacional. De acuerdo con Lepenies (1996), la sociología disputa un lugar entre las ciencias naturales, de un lado, y las ciencias humanas y la literatura, de otro.

En Francia la sociología se debatía frente a la literatura, en su propósito de ofrecer una orientación en la modernidad; ambas áreas adjudicándose un carácter científico. Fue apelando a la objetividad, al imitar las ciencias naturales en sus conceptos y búsqueda de leyes que la sociología gana legitimidad. Diferente de lo que ocurre en Alemania, donde las ciencias sociales estaban más próximas a la historia y su sentido interpretativo, de modo que no existía para la época una pretensión de erigirse como ciencia en los mismos términos que las ciencias "duras" (Ibíd.).

Es este contexto de largo plazo sociohistórico que, es preciso tener presente para enfrentarnos a los sentidos de los conceptos de cultura y valores en el pensamiento sociológico de los clásicos Durkheim y Weber. 


\section{Durkheim: valores ideales y civilización}

Durkheim usaba poco la palabra “cultura”. En la revista L'Année sociologique que funda en 1897, era traducida de textos foráneos como “civilización”. Una de las explicaciones para evitarla puede deberse a, como señala Cuche (2002, p. 31), una postura política de producción de conocimiento que apuntaba a diferenciarse del uso académico alemán.

En las condiciones del ámbito académico francés mencionadas, la forma en que Durkheim utiliza la noción de cultura refleja el uso del contexto nacional, en el que se refiere principalmente a la educación que se adquiere y se "cultiva" relacionada a un nivel de conocimiento. Revelando el proceso de "cultivar" como una acepción francesa de educarse, afirma que: "en los establecimientos en que realizan sus estudios los hijos de sabios, se encuentran en contacto con espíritus cultivados $^{25}$ o propios para recibir una elevada cultura, y la acción de ese medio nuevo no hace más que confirmar la del primero." (DURKHEIM, 1987, p.127). Ejemplos de esta acepción son: "cultura general” (DURKHEIM, 1987, p. 15), “cultura especializada” (Ibíd., p. 43), "cultura intelectual” (DURKHEIM, 2000, p. 34), "grado de cultura” (Ibíd., p. 134), “cultura inferior” (Ibíd., p. 335) y "cultura elevada".

De acuerdo con Durkheim, el sociólogo debería tener una buena formación en varias áreas, así, sugiere que "uma cultura psicológica, mais ainda que uma cultura biológica, constitui portanto para o sociólogo uma propedêutica necessária; mas ela só the será útil se ele libertar-se dela após tê-la recebido e a superar, completando-a por uma cultura especialmente sociológica" (DURKHEIM, 1978, p. 165). Además usa la concepción de cultura en un sentido general para referirse a la humanidad entera, con la variación de "cultura humana"26 (Op. Cit. 1987, p. 125; Op. Cit. 2000, p. 454).

\footnotetext{
${ }^{25}$ Añadí la cursiva.

26 "La impotencia progresiva de nuestra especie para producir razas nuevas ofrece incluso el más vivo contraste con la fecundidad contraria de las especies animales. ¿Qué significa esto sino que la cultura humana, a medida que se desenvuelve, es cada vez más refractaria a ese género de transmisión?" (DURKHEIM, 1987, p.125).
} 
Si bien Durkheim utiliza el término civilización más a menudo que el de cultura, en varios de sus textos acepta una visión de pluralidad de la civilizaciones al considerar el pensamiento conceptual y simbólico como característica de la unidad humana (CUCHE, 2002, p. 30). El significado con que distingue a la noción de civilización, constituye una serie de elementos simbólicos de la memoria, el conocimiento y la forma de sentir grupal de una sociedad específica. Para él, la civilización también tiene un nivel de desarrollo, al igual que la cultura, que es propio de cada sociedad. Asimismo la identifica en el ser humano como una cualidad ontológica, pues considera que "el hombre solo es un hombre porque es civilizado". (Durkheim, 2000, p. 395). En palabras de Durkheim:

Aquello que hace de nosotros seres verdaderamente humanos es precisamente lo que alcanzamos a asimilar de ese conjunto de ideas, sentimientos, creencias y preceptos de conducta que se llama civilización. [...] La civilización debe ser considerada como el conjunto de todos los bienes a los cuales atribuimos el más alto precio, la reunión de los más elevados valores humanos. Porque la sociedad es a la vez fuente y guardiana de la civilización. (DURKHEIM, 2006, p. 55).

Con la última frase de la cita textual, se demarca la diferencia entre sociedad, como el conjunto de individuos asociados, y civilización, que constituye una dimensión simbólica y espiritual de la sociedad. Así, su definición de cultura parece estar inmersa en la de civilización.

En la relectura del pensamiento de Durkheim, numerosos estudios encuentran en varios de sus términos un proyecto próximo a una teoría de la cultura. Algunos de ellos han sido las nociones de conciencia colectiva y representaciones colectivas (CUCHE, 2002; DA SILVA 2003; MURGUÍA, 2002), de imaginario social (MATOS, 2012), de moral (GÜELL, 2008), o de civilización, como ya expuse. Sin embargo, el clásico francés en su intento por refinar las herramientas teóricas, indica varias nociones que al igual que las características que define para la civilización, son un cúmulo de aspectos inmateriales, simbólicos e inmanentes a la sociedad y que, por consiguiente, tienen una significación cultural. Quiero referirme a dos de ellos: los valores y la moral. 
Según Durkheim, la sociedad tiene una primacía sobre sus integrantes, una capacidad de abarcarlos y excederlos. En ella se configuran conjuntos de ideales que son valores objetivos y buenos y que varían entre civilizaciones y épocas distintas. De ese modo, afirma que “los principales fenómenos sociales: religión, moral, derecho, economía, estética, no son otra cosa que sistemas de valores y por lo tanto, de ideales.” (DURKHEIM, 2006, p. 100). Dado que el valor de las cosas no es parte de su naturaleza intrínseca sino que se encuentra en la sociedad, que determina cuál es su valor apropiado o su ideal, los sujetos hacen juicios de valor en los que comparan las cosas con aspectos del ideal compartido. Los valores objetivos siempre son su referencia, puesto que "el respeto que ésta [la sociedad] inspira se comunica a las maneras de pensar, así como a las maneras de actuar a las que ella atribuye valor" (DURKHEIM, 2000, p. 528.).

No obstante, según Durkheim, los sujetos pueden hacer juicios de valor basados en concepciones o sensibilidades subjetivas diversas que no sean acordes a los ideales, es decir que según su perspectiva, pueden errar, puesto que solo los valores objetivos que construye la sociedad son los mejores. Por otra parte, el carácter objetivo de los valores no se refiere a un promedio que tiene la colectividad en sí, puesto que el autor señala que esta tiende a la mediocridad. Al respecto señala:

La diferencia es enorme entre la manera según la cual los valores son en realidad estimados por el individuo ordinario y esta escala objetiva de valores humanos sobre la que deben reglarse, en principio, nuestros juicios. La conciencia moral media es mediocre. No siente sino muy débilmente los deberes más elementales y por consiguiente, sus respectivos valores morales. (DURKHEIM, 2006, p. 85).

Esta compleja premisa se debe entender en el sentido de que tales valores son externos, existen fuera del individuo y al mismo tiempo se soportan en su colectividad. Adicionalmente, los ideales pueden cambiar con el tiempo, incluso llegar a debilitarse. Por ello, los sujetos deben interiorizarlos a través de ceremonias religiosas y laicas que cumplen el propósito de fortalecerlos y facilitar su aprehensión. Indica: 
Las predicaciones de toda clase llevadas a cabo por la Iglesia o por la escuela, las representaciones dramáticas, las manifestaciones artísticas; en una palabra, todo aquello que puede aproximar a los individuos y hacerlos comulgar en una misma vida intelectual y moral. Son algo así como débiles renacimientos parciales de la efervescencia de las épocas creadoras. (DURKHEIM, 2006, p. 96).

Por otra parte, los sistemas de valores tienen una conexión, aunque son de diferente tipo y no se pueden homologar entre ellos, el autor especifica que "una cosa es el valor económico y otra los valores morales, religiosos, estéticos, especulativos. Las tentativas frecuentemente hechas con el objeto de reducir unas a otras las ideas de bien, de belleza, de verdad y de útil, han sido siempre vanas." (DURKHEIM, 2006, p. 88).

Al igual que Weber, como explicaremos en la siguiente sección, Durkheim señala la importancia de una ciencia social que no tome partido de las evaluaciones: "Ela [a sociologia] os observa, os explica, mas não os julga; para ela, os fatos nada teriam de censurável. O bem e o mal não existem para ela. A ciência pode perfeitamente nos dizer de que maneira as causas produzem seus efeitos, não que finalidades devem ser buscadas”. (DURKHEIM, 1978, p. 39).

Entre los diferentes valores, los más relevantes son los morales, cuestión capital en toda la obra durkhemiana. Él concibe la moral como un conjunto de normas de conducta que provienen de la autoridad de la sociedad que los sujetos obedecen por un sentido del deber y que al tiempo, tales normas se presentan como atrayentes y buenas, y así suscitan el deseo de obedecerlas en los individuos. El deber y la deseabilidad, son pues, sus dos características básicas. En el estudio de las religiones, Durkheim identifica a lo sagrado como una abstracción de la sociedad y de sus ideales. Así, el concepto de sociedad es inseparable de la idea de lo moral, ambos están imbricados. Y lo sagrado o la sociedad, es aquello que es inviolable, amado (DURKHEIM, 2000, p. 37).

Ahora bien, para Durkheim puede haber reglas de comportamiento específicas en diferentes ámbitos que los individuos sopesan para orientar su actuar. Empero, una acción es moral solo cuando el sujeto se hace consciente de su 
responsabilidad con los otros, y se guía por un valor que tiene por objeto a la colectividad. Él expone:

Pero si no podemos estar vinculados por el deber sino a sujetos conscientes, después que hemos eliminado todo sujeto individual, no queda otro fin posible a la actividad moral que el sujeto sui generis formado por una pluralidad de sujetos individuales asociados de manera de formar un grupo, es decir, no resta sino el sujeto colectivo. (DURKHEIM, 2006, p. 52).

Ante una aparente discordancia entre el deber y deseo Durkheim argumenta a sus detractores que no se contraponen en la medida en que el sujeto sea un sujeto moral y consciente que crea en la superioridad moral de la sociedad. Pues el sujeto no rechaza las normas objetivas, sin negarse a sí mismo.

Al argumentar de esta manera la existencia de unos valores sublimes que reposan en el colectivo y el deseo de comunión con ellos de los individuos, se nota como sirven a modo de una fuerza de cohesión social.

Dada la exterioridad de los valores morales objetivos, Durkheim propone que se debe educar y socializar a los individuos en ellos. Con esta conclusión se traslada a los dominios de la pedagogía y la política en la que adquiere un matiz prescriptivo y la compromete con un papel de intervención social que debe ser asumido por la guía del Estado. Así, además de sus funciones políticas el Estado se torna en un agente moral (OLIVEIRA, 210, p. 126). Ahora bien, escapa a los objetivos de este ensayo el camino trazado por Durkheim para una pedagogía de la moral y el estudio de la falta de cohesión social o anomia.

\section{Weber: significación y ethos}

La noción de cultura es recurrente en la obra weberiana, dada su relevancia comentada para el contexto alemán. Se puede identificar cierta polisemia en su uso que no solo está en sus obras, sino que hace parte de la variedad de significados con la que ha cargado la palabra "cultura" en la historia de las ciencias sociales (CUCHE, 2002). El uso como adjetivo resalta más que como sustantivo en la obra weberiana. Uno de los sentidos de cultura a los que se refiere el autor, quizá el más sobresaliente, es conceptualizado como un grupo humano en 
un lugar y espacio concretos, o "históricamente dado" cuyos miembros comparten tradiciones y significados particulares. Puede tratarse de poblaciones o sociedades localizadas en distintas épocas y de escala media o macro. Algunos ejemplos de ello, extraídos del que se considera su libro más importante, Economía y sociedad (1964), son "cultura moderna" (p. 732; p. 1138), "cultura china” (p. 792), “culturas desarrolladas” (p. 754), “cultura antigua” (p. 797; p. 1173) “cultura fenicia” (p. 841), "cultura francesa y holandesa", en otro texto (WEBER, 1991, p.47). Respecto a un ámbito más abarcador, escribe "desarrollo cultural de Oriente y Occidente" (Op. Cit., p. 892).

Otro sentido que le adjudica se refiere a diferentes ámbitos del conocimiento, o a lo que llama contenidos de la cultura. Explica "no se trata de la relación de la economía con los dominios -con los contenidos- particulares de la cultura (literatura, arte, ciencia, etc.)" (Ibíd., p. 289). Otros ejemplos del sentido anterior, son “cultura material” (1964, p. 854), “cultura técnica” (Ibíd., p. 734) "cultura espiritual" (Ibíd., p. 780) “cultura musical” (Ibíd., p. 1150).

Entre ellos, resalta la alusión a la cultura como la formación intelectual y artística en una acepción similar a la francesa. La frase siguiente lo evidencia: "La enseñanza del "especialista" como factor destacado en la cultura, solo el Occidente los ha forjado" (WEBER, 1991, p. 33).

A pesar de los diversos usos, el autor hace un esfuerzo por definirla y erradicar sus ambigüedades al vincularla al objeto científico. En Ensayos de metodología sociológica (1958), usa la expresión ciencias sociales y ciencias culturales como sinónimos.

Explica:

La ciencia social que queremos promover es una ciencia de realidad. Queremos comprender la realidad de la vida que nos circunda, y en la cual estamos inmersos, en su especificidad; queremos comprender, por un lado, la conexión y significación cultural de sus manifestaciones individuales en su configuración actual, y, por el otro, las razones por las cuales ha llegado a ser históricamente a ser así y no de otro modo (WEBER, 1958, p. 61).

Más adelante repite que la denominación de ciencias de la cultura se refiere a campos de estudio que pretenden dar cuenta de las manifestaciones 
sociales en su significación cultural (WEBER, 1958, p. 65). La palabra clave para comprender la definición de cultura que propone, es "significación", y la encontramos de nuevo en su definición: “'Cultura' es una sección limitada de la infinitud desprovista del sentido del acaecer universal, a la cual los seres humanos otorgan sentido y significación” (WEBER, 1958, p. 70).

Sin embargo, el proceso de atribución de significado cultural conlleva siempre una forma de valorar esa realidad dinámica; el sujeto otorga un sentido y evalúa lo que logra capturar de ella por medio de conjuntos de valores diversos. Así, la idea de cultura se vuelve indisociable del proceso valorativo por el que se construye, y ambos elementos se vislumbran como nociones centrales que atraviesan el análisis de toda su obra, como resalta Karlberg (2010, p. 32). Su perspectiva de la cultura anticipa lo que más tarde interesará a la antropología interpretativa, que Geertz basado en él reelabora al entender la cultura como una trama de significados compartidos (PASSIANI, 2001, p. 53).

Weber profundiza en la reflexión epistemológica, y señala que la ciencia debe evitar hacer juicios de valor en vista de acercarse a un conocimiento objetivo. El científico social debe tratar de aislar sus formas de valorizar en la medida de lo posible, aunque sea consciente de que también su acción se guía por evaluaciones. La misma elección del objeto de estudio implica que se ha considerado que unos temas son más importantes que otros. La ciencia debe entonces, dilucidar las condiciones en las que se emiten y conforman tales valoraciones, estudiar la causalidad que determina ciertas configuraciones de valor y su incidencia en la acción social, que es aquella que "se orienta por las acciones de otros" (WEBER, 1964, p. 18). Sin embargo, la ciencia sí puede ayudar al humano a ser coherente en sus deseos e ideas al explicar la lógica de los medios y fines y al anular toda contradicción en el proceso.

Para Weber, la sociología podría explicar la causalidad de los significados culturales de la acción por medio de la comprensión racional -intelectual- y la comprensión intuitiva o empática -emocional- (KARLBERG, 2010, p. 34). La comprensión de la causalidad de las uniformidades, se puede dar bajo la caracterización de los tipos ideales, abstracciones de la realidad diseñadas a partir 
de varias perspectivas del mismo objeto, que constituyen las características ideales de un fenómeno social. Así un tipo ideal representa un modelo conformado por valores arquetípicos de una cultura particular. Organiza cuatro tipos de un rango de máximo racionalismo a menor irracionalismo: racional con arreglo a fines, racional con arreglo a valores, afectiva, y tradicional (WEBER, 1964, p. 20). Aunque solo el segundo de ellos le da mayor peso a los valores, todos constituyen tomas de posición valorativas (GARRIGUE, 2009, p. 48) que adjudican relevancia a un aspecto o a otro, aunque las motivaciones varíen en su grado de racionalidad.

Si para Weber, la cultura es la parte de la realidad que se significa, esto se hace mediante un proceso valorativo racional o irracional según los cuatro conceptos típicos ideales que propone, en el que se ponen en práctica conjuntos de valoraciones de diversos tipos: estéticos, éticos, morales, económicos, religiosos, científicos, entre otros. Sin embargo la cantidad diversa de sistemas de valores tienden a confrontarse y chocar entre sí en diferentes esferas culturales grupales o individuales, como señala Freund (2003, p. 24) "O antagonismo dos valores é simplesmente insuperável". Él lo explica en varios apartados: "La religión de fraternidad ha estado siempre en antagonismo con las órdenes y valores mundanos y este antagonismo se ha agudizado tanto más cuanto más firmemente se han puesto en práctica sus exigencias". (WEBER, 1987, p. 57). De la misma manera los valores actúan en contraposición dialéctica entre las ideas de racionalización e irracionalización tan relevantes en su obra.

Nunca uma coisa é "irracional" em si, mas sempre de um determinado ponto de vista "racional': Para quem é irreligioso, toda conduta de vida religiosa é "irracional", assim como para o hedonista é irracional toda conduta de vida ascética, por mais que, levando-se em conta o valor último de cada qual, se trate de uma "racionalização". Se o presente ensaio tiver que contribuir para algo, que seja para por a descoberto em sua polivalência o conceito apenas aparentemente unívoco de "racional". (WEBER, 2004, p. 175).

En La ética protestante y el espíritu del capitalismo (2004), una de sus obras más conocidas, estudia el proceso de racionalización de Occidente y encuentra en la resignificación de valores ético-religiosos a valores ético-profanos 
una de las causas del principal sistema económico moderno. Él hace un análisis de los fundamentos calvinistas en sus textos más importantes y evidencia cómo valores del ethos religioso -como el ascetismo, la búsqueda de lucro y el trabajo como vocación- que prescriben algunas vertientes del protestantismo, se trasladan al ámbito secular desligados de la religiosidad y devienen en valores culturales y laborales, incidiendo en la conducta social que incentivaría el desarrollo capitalista (WEBER, 2004).

El autor define ethos como "um determinado estilo de vida regido por normas e folhado a ética" (WEBER, 2004, p. 51). De acuerdo al argumento del texto, deducimos que ética o ethos, para Weber es un conjunto de valores y máximas que sus agentes consideran positivos o buenos, que orientan su comportamiento en ciertos ámbitos de una cultura, en determinado contexto sociohistórico. Evidencia de ello es que al igual que el concepto de cultura, cuando la palabra "ética" aparece ligada a un adjetivo, la forma de comportamiento se restringe a ese ámbito, por ejemplo, "ética religiosa medieval” (Ibíd., p. 77), “ética do calvinismo" (Ibíd., p. 79) y “ética profissional” (Ibíd., p. 124); o "doctrina ética”, que sería un conjunto de reglas que prescriben la conducta práctica para lograr una buena conducta (Ibíd., p. 51).

Dada la importancia de las evaluaciones valorativas que Weber identifica en el actuar de los individuos, el científico, de igual manera, asume tomas de posición valorativas al seleccionar su objeto de estudio (WEBER, 1958, p. 131). Para el cásico alemán el individuo es el que elige cómo orientar su acción social. Weber enfatiza en la pluralidad de motivos lo que distingue su sociología de las que consideran la estructura social como el determinante principal, entre ellas la durkhemiana. No obstante esto le hacer tropezar con un problema, como señala Karlberg; su desarrollo teórico plantea el siguiente interrogante "por que razões subjetivas as pessoas orientam sua ação conjunta tal como formulada por grupos definidos?” (KARLBERG, 2010, p. 37). Si bien no es propósito de este ejercicio extendernos en una posible respuesta, un aspecto clave para entender esta dicotomía del individuo y el grupo, radica en que, aunque el sujeto tiene 
libertad de elección en su forma de actuar, se desempeña en una sociedad que orienta y regula su acción en varios ámbitos por medio de normas o valores establecidos de significación positiva y culturalmente compartida. De ello es ejemplo el siguiente párrafo en que observa el medio opresivo capitalista:

\begin{abstract}
Atualmente a ordem econômica capitalista é um imenso cosmos em que o individuo já nasce dentro e que para ele, ao menos enquanto individuo, se da como um fato, uma crosta que ele não pode alterar e dentro da qual tem que viver Esse cosmos impõe ao individuo, preso nas redes do mercado, as normas de ação econômica (WEBER, 2004, p. 47-48).
\end{abstract}

De esta manera las formas de valorar no solo permiten al individuo relacionarse con el mundo, sino que también se vislumbran como procesos de articulación entre individuo y sociedad que permean y significan sus acciones. $Y$ es en este punto donde converge con la concepción durkhemiana de moral.

\title{
Convergencias y desencuentros
}

Expuestas las concepciones de Durkheim y Weber en lo relativo a la cultura y a los valores en las líneas anteriores, paso a señalar algunas convergencias entre ellas y sus puntos de desencuentro que vine esbozando a lo largo de la exposición.

Tanto Durkheim como Weber usan la noción de cultura como se desenvolvía en sus respectivos contextos nacionales. Para el primero, con un énfasis en un proceso que se adquiere mediante la educación, y también como un elemento constitutivo de la humanidad. Para el segundo, en una dirección particularista, aludiendo a un grupo humano históricamente dado, o a ciertos ámbitos de la cultura. Ahora bien, ambos científicos profundizan en ella y aseveran la importancia simbólica de la acción social. Weber va más allá del uso convencional de su época al afirmar que la cultura es el proceso por el cual significamos una parte de la realidad. La traducción deliberada del término de cultura por el de civilización que hace Durkheim, se refiere a grupos sociales específicos en el sentido particularista alemán. Por su parte, al desarrollar la 
noción de civilización, afirma que ella se compone por un conjunto de valores sublimes, que comprende a la moral, y también a la cultura en la acepción francesa. Además, destaca su característica ontológica en el ser humano, pues para él no existe humano no civilizado.

Si observamos el tratamiento de los valores en las teorías de los dos sociólogos podemos señalar puntos de encuentro de peso que los ligan con la cultura. Para ambos, los valores y otros aspectos inmateriales y significativos yacen en el colectivo; los individuos confieren un sentido a la realidad con referencia al colectivo, aunque cada sujeto pueda diferir de ellas. Son culturales, en otras palabras, son valores particulares a cada grupo humano.

Cuando Durkheim afirma que "hay así una región de la naturaleza donde la fórmula del idealismo se aplica casi al pie de la letra: es el reino social. La idea constituye allí, mucho más que en otra parte, la realidad" (DURKHEIM, 2000 , p. 329), resalta que lo que caracteriza a la forma de relación de los sujetos con la realidad son las ideas que se hacen de ellas y que residen en la sociedad, en el conjunto de sujetos, ideas que son un conocimiento que posee valor objetivo dado su origen social, no natural. Estos abordajes que comprenden a la sociedad como una matriz de significaciones, valores y otras formas inmateriales y compartidas, convergen con el planteamiento de Weber, para el que la cultura es el proceso valorativo que nos permite asignar una significación a la caótica realidad. Así en ambos sociólogos, proceso valorativo y atribuciones significativas son indisociables e inmanentes a los sujetos y a su articulación con la colectividad.

No obstante, las concepciones de ambos autores abrigan diferencias. Para ambos, existen varios sistemas de valores que son particulares a cada grupo humano, y que pueden cambiar con el tiempo; unos valores son colectivos y otros individuales. Entre los diferentes valores, los valores morales son los más importantes según Durkheim. Son valores objetivos, verdaderamente buenos y de acuerdo a ello, los individuos deben esforzarse por aprehenderlos, y al mismo tiempo desean hacerlo. Dado que lo valores pueden debilitarse es necesario reforzarlos con los rituales, y con otras propuestas pedagógicas y políticas en las 
que el investigador adquiere una postura más activa con una ciencia social aplicada. De esta manera el programa teórico del clásico francés hace énfasis en la sociedad como fuente de toda idea objetiva y buena de la realidad. Mientras que para Weber, el sujeto puede orientarse por formas de valoración disímiles a las objetivas, su explicación entiende al individuo como con un ser con mayor rango acción y de posibilidades de agencia. La idea de la moral, no constituye el centro de sus investigaciones, y no hace una correlación teórica entre el deber y la deseabilidad, por ello el deseo de lo bueno según su pensamiento no explica la acción social, ni la búsqueda de una armonía colectiva. Por otra parte, otras nociones que utiliza son más útiles para explicar la acción social.

Para Weber, el ethos consiste en normas de comportamiento de determinados ámbitos culturales que se organizan según una serie de valores relativos a una cultura dada. Así, analizar cómo se configuran y cambian los valores históricamente y son llevados a la práctica por actores que siguen una ética, puede explicar la causa de ciertos fenómenos, como él lo hace con el capitalismo.

Como pioneros de la disciplina, tanto Durkheim como Weber declararon aspectos capitales para la formación de un científico social aún vigentes. Ellos coinciden en que la ciencia social no debe hacer juicios de valor sobre su objeto, aunque las valoraciones tienen una relevancia significativa por cuanto definen la relación de los sujetos con la realidad y son parte de las configuraciones culturales. En cambio, la ciencia sí puede ayudar a la sociedad a alcanzar la claridad en sus formas de valorar al indicarle un camino lógico en el que vislumbre el orden de sus proposiciones que dé cuenta de su causalidad y anule toda contradicción (DURKHEIM, 2006, p. 100; WEBER, 1987, p. 43).

Como vimos, los cuatro tipos ideales de la acción social son tomas de posición valorativas, aunque solo uno de ellos tiene como móvil a los valores. Weber advertía que el desarrollo de Occidente estaba cada vez más dirigido a lo que explicaba con uno de sus tipos ideales, como una forma de actuar racional con relación a fines, esto es, hacia una racionalización creciente del mundo occidental. Sin embargo, dada la lucha antagónica de valores en permanente dialéctica que él mismo señalaba en su teoría, fue pesimista al no considerar la posible 
remagificación o reecantamiento de Occidente, que plantea una tarea por estudiar.

En ese sentido, si nos basamos en la hipótesis que afirman de que existe una simultaneidad de varios sistemas de valores de carácter dicotómico, es decir, tanto subjetivos como objetivos, es visible la semilla de un pensamiento complejo en ambos sociólogos sobre la diversidad cultural ampliamente reconocida en la contemporaneidad.

En la actualidad, el desafío del estudio sociológico se vuelve mayor dados los contextos multiculturales y globalizados que, aunque no fueron objeto de las concepciones de los sociólogos clásicos, su pensamiento sienta una base para tal abordaje. En tal medio, es relevante inquirir de qué manera se configura una multiplicidad de sistemas de valor en pugna, conjuntos de valores objetivos que parten de colectividades diversas y comprender cómo los agentes los defienden, los establecen o los resignifican. Indagar sobre los sistemas de valores nos puede guiar en la comprensión de los sentidos culturales y sus geopolíticas en las sociedades contemporáneas.

\section{Referencias}

CUCHE, Denys. O Conceito de Cultura nas Ciências Sociais. Tradução de Viviane Ribeiro. 2. ed. Bauru: EDUSC, 2002.

DA SILVA, Erlando. Do conhecimento sociológico à teoria das representações sociais. Sociedade e Cultura. Goiânia: UFG, v. 6, n. 2, julho 2003, p. 189-199. DURKHEIM, Emile. As Regras do Método Sociológico. São Paulo: Abril, 1978. La División del Trabajo Social. Traducción de Carlos G. Posada. 2. ed. Madrid: Akal, 1987. Las formas elementales de la vida religiosa. México D.F.: Colofón, 2000. Sociología y filosofía. Traducción de José María Bolaúo. José Luis Monereo (Ed.). Granada: Comares, 2006.

ELIAS, Norbert. 0 processo civilizador. 2. ed. Rio de Janeiro: Jorge Zahar Ed., 1994

FREUND, Julien. Sociologia de Max Weber. Tradução de Luís Claudio de Castro. Rio de Janeiro: Forense-Universitária, 5a ed. 2003.

GARRIGUE, Olivier. Sociología del valor: valores individuales y valores colectivos. Análisis sociológico y síntesis de un modelo teórico. $124 \mathrm{f}$. Tesis (Maestría en Ciencia política y sociología) FLACSO, Buenos Aires, 2009. 
GÜELL, Pedro. ¿Qué se dice cuando se dice cultura? Notas sobre el nombre de un problema. Revista de Sociología. Santiago de Chile, Universidad de Chile n. 22, 2008. p. 37-64.

KARLBERG, Stephen. Max Weber: Uma introdução. Rio de Janeiro: Zahar, 2010. LEPENIES, Wolf. As três culturas. São Paulo: Edusp, 1996.

MATOS, J. El problema de la sociología y el imaginario social en el pensamiento clásico y contemporáneo. Contribuciones a las Ciencias Sociales, Enero 2012, Disponível em: <www.eumed.net/rev/cccss/17/> Acesso em: 20 jun. 2014. MURGUÍA, Adriana. Durkheim y la cultura. Una lectura contemporánea. Sociológica. México D.F., an. 17, n. 50, septiembre 2002, p. 83-102.

OLIVEIRA, Márcio de. O Estado em Durkheim: elementos para um debate sobre sua sociologia política. Revista de Sociologia e Política, v. 18, n. 37, 2010, p.125-135. PASSIANI, Ênio. Max Weber: um pensador da cultura. Dialogia, São Paulo, Outubro de 2001, p. 47-55.

PIERUCCI, Antônio. Apresentação. In: WEBER, Max. A ética protestante e o espíritu do capitalismo. Tradução de Marcos Mariani de Macedo. São Paulo: Companhia das Letras, 2004.

WEBER, Max. Ensayos sobre metodología sociológica. Traducción José Luis Etcheverry. Buenos Aires: Amorrortu, 1958.

Economía y sociedad, Esbozo de sociología comprensiva. Traducción de José Medina Echavarría, Juan Roura Farella, Eugenio Ímaz, Eduardo García Maynez y José Ferrater Mora. 2 Ed. México, D.F: Fondo de Cultura Económica. 1964. - Ensayos sobre sociología de la religión. Madrid: Taurus, 1987. A ética protestante e o espíritu do capitalismo. Tradução de Marcos Mariani de Macedo. São Paulo: Companhia das Letras, 2004.

Recebido: 06 jan. 2019 Aceito: 22 jan. 2019 